\title{
Reversible Paclitaxel-Induced Bilateral Vocal Fold Paresis
}

\author{
Jeffrey Hsu, Melin Tan-Geller \\ Montefiore Medical Center, New York, USA \\ Email: jeffreyvhsu@gmail.com
}

Received 26 March 2015; accepted 18 May 2015; published 21 May 2015

Copyright (C) 2015 by author and Scientific Research Publishing Inc. This work is licensed under the Creative Commons Attribution International License (CC BY). http://creativecommons.org/licenses/by/4.0/

(c) (i) Open Access

\begin{abstract}
Introduction: Chemotherapy is a rare cause of iatrogenic vocal fold dysfunction. It has been reported in three main classes of chemotherapy agents and often occurs during the treatment interval. We present a case of bilateral vocal cord paresis with delayed presentation after completion of chemotherapy. Methods: One case, managed with observation and serial exams, is presented. A review of previous case reports of chemotherapy-induced vocal cord paresis and possible mechanisms of injury was performed. Results: Patient improved both symptomatically and through objective findings over the one-year course of observation. Conclusion: Diagnosis of chemotherapy-induced vocal cord paresis is dependent on a thorough history and physical exam. Management is predicated in that the dysfunction is often dose dependent and reversible, necessitating both cessation of the offending agent and the knowledge that any treatment is likely needed for only a temporary time. Chemotherapy-induced vocal fold paresis should be in the differential for patients presenting with hoarseness, dysphonia, stridor and a positive chemotherapy history.
\end{abstract}

\section{Keywords}

Vocal Cord Paralysis, Chemotherapy

\section{Introduction}

The most common cause of vocal fold paralysis is iatrogenic injury from surgical intervention or intubation. Thyroid and parathyroid surgery as well as cardiothoracic surgery defines a large percentage of surgical vocal fold paralysis etiology. A much less common iatrogenic source of injury is chemotherapy. While several chemotherapeutic drugs such as vincristine and vinblastine are known to be neurotoxic, few are known to affect vocal fold function.

Paclitaxel (Taxol) is a common chemotherapeutic drug originally developed from the Pacific yew tree (Taxus 
brevifolia) and has been used in the treatment in multiple malignancies including lung, ovarian, and breast cancers. While neurotoxicity is its biggest limit in application, currently there is only one case of vocal fold palsy reported in the literature to date [1]. It was diagnosed during active treatment and found to be reversible upon cessation of drug administration. We presented a case of delayed presentation of bilateral vocal fold paresis in a patient who completed a neo adjuvant chemotherapy regimen including paclitaxel for breast cancer. It was the second case of its kind, but distinguishable in that onset of symptomatic vocal fold paresis starting well after completion of treatment.

\section{Case Report}

A 62-year-old female with history of left invasive ductal carcinoma presented in April 2013 with several months of loss of the high vocal range and increased vocal strain. She is a classically trained singer who sings in a choir and at that time, noticed decreased range in her highest singing frequencies with need for increased straining. She denied any issues with her speaking voice, dysphagia, stridor or respiratory difficulty. Her oncologists noted her to have grade 1 fatigue and neuropathy, manifesting as upper extremity weakness and lower extremity paresthesias.

She underwent 12 treatments of neoadjuvant therapy of Paclitaxel, Herceptin and Vorinostat for her clinically staged cT3N3MO breast cancer from July 2012 to October 2012. Besides an episode of febrile neutropenia, no complications were described. Further treatments for her breast cancer included left mastectomy with a negative axillary lymph node dissection in January 2013 and subsequent intensity modulated radiotherapy along with maintenance Herceptin.

Her initial otolaryngologic exam was significant for mobile but paretic vocal folds bilaterally and near complete glottic closure. She was treated with multiple sessions of voice therapy where she learned exercises on how to compensate for her vocal fold paresis. Repeat endoscopic exam two months later revealed full mobility of the left fold with residual mild paresis of the right true fold and complete glottic closure (Figure 1). One year after her initial presentation, she was without voice complaints and laryngoscopy revealed completely normal vocal fold mobility.

As is the custom in our institution, no signed informed consent was necessary due to the deidentification of the patient's exam and clinical course but verbal consent was obtained from the patient prior to publication.

\section{Discussion}

Vocal fold paresis often presents with symptoms such as dysphonia, hoarseness, dysphagia or aspiration. The four most common etiologies have been cited to be malignancy, iatrogenic, idiopathic, and neck trauma [2]. Common iatrogenic causes include surgeries in the head and neck region as well as endotracheal intubations. One less common iatrogenic cause is neurotoxicity from chemotherapeutic agents.

Neurotoxicity is a common side effect of chemotherapy drugs, mostly seen with vinca alkaloids and taxanes. Historically, the dose limiting effects have been peripheral neuropathies but have also been known to affect the motor and autonomic nervous systems. Symptoms typically manifest with increased dose dependency, increased infusion rates and with onset within close proximity of consumption. Cranial nerve palsies have been a very rare presentation of chemotherapy-induced neurotoxicity [3]-[5]. Though rare, there are at least three different chemotherapeutic classes known to cause vocal fold paresis.

Since its introduction, there have been over 43 case reports of vinca alkaloids induced vocal fold paresis [5][9]. Vinca alkaloids function through prevention of tubulin polymerization, disrupting microtubule formation and halting cell division. Though the pathophysiology of neurotoxicity has not been clearly defined, it is hypothesized that the vinca alkaloids disrupts axonal transport and nerve conductance. Clinical manifestations have included hoarseness, dysphagia, cough, and stridor with patients being diagnosed with both unilateral and bilateral vocal fold paresis. Vincristine has been found to be the most neurotoxic, followed by vinblastine, and vinorelbine [10]. In the Pediatric Otolaryngology Group study, four cases of vincristine-induced laryngeal paralysis were seen over five and half years among 293 children. In their literature search, only 10 pediatric cases had been previously reported since 1966, likely revealing an underreporting of cases [11]. Though symptoms are often reversible with cessation of use, patients can undergo a phenomenon called coasting where symptoms worsen before they improve [5] [10]. Treatment has ranged from observation to tracheotomy to address airway compromise. 


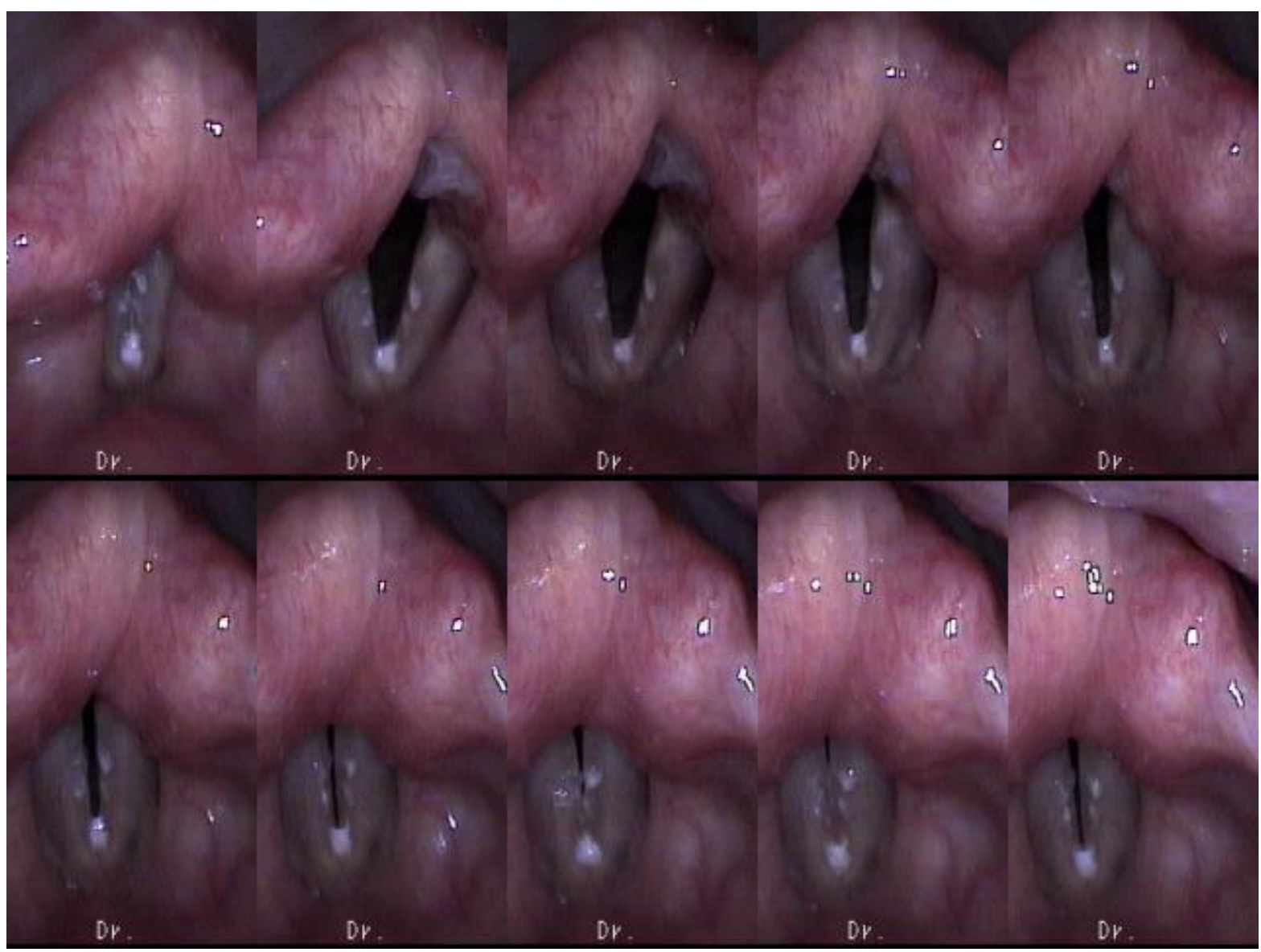

Figure 1. Fiberoptic laryngeal exam. Sequential still images of patient's fiberoptic laryngeal exam during one of the serial follow up exams. From right to left, the left vocal fold is seen to be in varying position while the patient is abducting and adducting her vocal fold while the right vocal fold remains in similar position throughout the exam. Here, we are able to see the partial recovery of the left vocal fold while the right is still paretic.

Cisplatin is a platinum derivative who mechanism involves the inhibition of DNA synthesis by forming crosslinks of DNA molecules. Its neurotoxic effects normally affect the sensory system and occur with accumulating doses. In most patients it is often reversible, though incomplete [10]. Again, while the exact neuropathic mechanism is not known, it is hypothesized that injury occurs at the dorsal root ganglion for sensory nerves while also causing axonopathy in other nerve types. There has been one previously cited case involving a patient receiving cisplatin for small cell lung cancer presenting with stridor and bilateral vocal fold paralysis [12]. The patient required an urgent tracheotomy but was ultimately able to bedecannulated with return of vocal fold function.

Paclitaxel is a part of many chemotherapy regimens for a wide range of cancers. Its chemotherapeutic effects are due to its ability to promote the formation and stabilization of polymerized microtubules, disrupting cell mitosis. [10] It, like many other chemotherapeutic drugs, is limited by neurotoxicity. This manifests itself most often as a sensory peripheral neuropathy in a stocking-glove presentation [10], but has also been reported to cause other, more unusual presentations including vocal fold palsy [1] and facial nerve palsy [3]. With paclitaxel, neurotoxic symptoms usually manifest in first $24-72$ hours [13], which are dose dependent [14], and often associated with prior neurotoxic agents and underlying neuropathy [13] [14].

There has been one reported case of paclitaxel induced vocal fold paresis. The patient developed unilateral vocal fold paresis after her 3rd dose of paclitaxel which was reversed upon cessation of the drug [1]. Paclitaxel binds to the B-tubulin subunit, preventing of microtubule disassembly which leads to inability for a cell to divide and then its death. Like the vinca alkaloids, its toxic effects have been postulated though impairment of axoplasmic transport via its disruption of normal microtubule function [10]. In our case, like the previously cited 
cranial nerve neuropathies [3], neurotoxicity was reversible. Most instances of neuropathy have showed recovery within six months of drug cessation.

In contrast to the previously described case involving paclitaxel, our patient presented nearly six months after the completion of therapy as opposed to during the course of receiving paclitaxel. On exam, both vocal folds appeared paretic compared to the previously seen unilateral injury. Our patient's symptoms resolved with vigilant observation and no further treatment. It appears that from comparisons to the previously cited case, there can be a varied manifestation of paclitaxel-induced toxicity of the recurrent laryngeal nerve in both respects to time of presentation and clinical findings. This could imply that like vinca associated toxicity, many other patients might have undiagnosed paclitaxel mediated vocal fold injury and this phenomenon is underreported [11].

Other known neurotoxic chemotherapeutic drugs include ixabepilone, bortezomib, thalidomide, eribulin, nelarabine, cytarabine, procarbazine, and teoposdie. Though there have been no reports of vocal fold paresis with their use, patients on any of these chemotherapeutic drugs have the possibility of recurrent laryngeal nerve injury.

In our case, management of the bilateral vocal cord paresis was conservative, with the use of voice therapy and serial follow up. From the previously reported literature cases, patients with vocal fold paresis treatment ranged from observation to tracheotomy [5] [10] [11]. Ultimately, the magnitude of intervention is correlated to the level of airway obstruction and functional deficits that result from the vocal fold dysfunction.

\section{Conclusion}

While trauma, malignancy, and iatrogenic factors are common causes of vocal fold paresis, history of neurotoxic chemotherapeutic drugs should be part of any evaluation. This importance lies in the experience that it is often dose dependent and reversible, necessitating both cessation of the offending agent and the knowledge that any treatment is likely needed for only a temporary time. Though it is a diagnosis of exclusion, chemotherapy-induced vocal fold paresis should be in the differential for patients presenting with hoarseness, dysphonia, stridor and a positive chemotherapy history.

\section{Conflict of Interest}

The authors declare that they have no conflict of interest.

\section{References}

[1] Choi, B.S. and Robins, H.I. (2008) Reversible Paclitaxel-Induced Vocal Cord Paralysis with Later Recall with Vinorelbine. Cancer Chemotherapy and Pharmacology, 61, 345-346. http://dx.doi.org/10.1007/s00280-007-0453-4

[2] Benninger, M.S., Gillen, J.B. and Altman, J.S. (1998) Changing Etiology of Vocal Fold immobility. The Laryngoscope, 108, 1346-1350. http://dx.doi.org/10.1097/00005537-199809000-00016

[3] Lee, R.T., Oster, M.W., Balmaceda, C., Hesdorffer, C.S., Vahdat, L.T. and Papadopoulos, K.P. (1999) Bilateral Facial Nerve Palsy Secondary to the Administration of High-Dose Paclitaxel. Annals of Oncology, 10, 1245-1247. http://dx.doi.org/10.1023/A:1008380800394

[4] Lash, S.C., Williams, C.P.R., Marsh, C.S., Crithchley, C., Hodgkins, P.R. and MacKie, E.J. (2004) Acute Sixth-Nerve Palsy after Vincristine Therapy. Journal of American Association for Pediatric Ophthalmology and Strabismus, 8, 6768. http://dx.doi.org/10.1016/j.jaapos.2003.07.010

[5] Abdul Latiff, Z., Azlin Kamal, N., Jahendran, J., Alias, H., See Goh, B., Zulkifli Syed Zakaria, S., et al. (2010) Vincristine-Induced Vocal Cord Palsy: Case Report and Review of the Literature. Journal Pediatric Hematology Oncology, 32, 407-410. http://dx.doi.org/10.1097/MPH.0b013e3181e01584

[6] Burns, B.V. and Shotton, J.C. (1998) Vocal Fold Palsy Following Vinca Alkaloid Treatment. The Journal of Laryngology and Otolaryngology, 112, 485-487.

[7] Ahmed, A., Williams, D. and Nicholson, J. (2007) Vincristine-Induced Bilateral Vocal Cord Paralysis in Children. Pediatric Blood \& Cancer, 48, 248. http://dx.doi.org/10.1002/pbc.20850

[8] Anghelescu, D., De Armendi, A.J., Thompson, J., Sillos, E.M.A., Pui, C.-H. and Sandlund, J. (2002) Vincristine-Induced Vocal Cord Paralysis in an Infant. Paediatric Anaesthesia, 12, 168-170. http://dx.doi.org/10.1046/j.1460-9592.2002.00816.x

[9] Bacon, L.C., Barnett, M.J. and Abou Mourad, Y.R. (2012) Vincristine-Induced Vocal Cord Paralysis in a Patient with Acute Lymphoblastic Leukemia. Annals of Hematology, 91, 971-972. http://dx.doi.org/10.1007/s00277-011-1348-3 
[10] Miltenburg, N.C. and Boogerd, W. (2014) Chemotherapy-Induced Neuropathy: A Comprehensive Survey. Cancer Treatment Reviews, Published Online. http://dx.doi.org/10.1016/j.ctrv.2014.04.004

[11] Kuruvilla, G., Perry, S., Wilson, B. and El-Hakim, H. (2009) The Natural History of Vincristine-Induced Laryngeal Paralysis in Children. Archives of Otolaryngology and Head and Neck Surgery, 135, 101-105. http://dx.doi.org/10.1001/archoto.2008.514

[12] Taha, H., Irfan, S. and Krishnamurthy, M. (1999) Cisplatin Induced Reversible Bilateral Vocal Cord Paralysis: An Undescribed Complication of Cisplatin. Head and Neck, 21, 78-79. http://dx.doi.org/10.1002/(SICI)1097-0347(199901)21:1<78::AID-HED11>3.0.CO;2-7

[13] Lipton, R.B., Apfel, S.C., Dutcher, J.P., Rosenberg, R., Kaplan, J, Berger, A., Einzig, A.I., Wiernik, P. and Schaumburg H.H. (1989) Taxol Produces a Predominantly Sensory Neuropathy. Neurology, 39, 368-373. http://dx.doi.org/10.1212/WNL.39.3.368

[14] Postma, T.J., Vermorken, J.B., Liefting, A.J.M., Pinedo, H.M. and Heimans, J.J. (1995) Paclitaxel Induced Neuropathy. Annals of Oncology, 6, 489-494. 